\title{
A BLOWUP CRITERION FOR THE FULL COMPRESSIBLE NAVIER-STOKES EQUATIONS*
}

\author{
XIANGDI HUANG ${ }^{\dagger}$
}

Abstract. In this paper, we establish a blow up criterion for strong solutions of the full compressible Navier-Stokes equations just in terms of the gradient of the velocity. It shows that the gradient of the velocity alone dominates the global existence of strong solutions.

Key words. Blowup, full compressible Navier-Stokes.

AMS subject classifications. 35Q30, 76N10

1. Introduction. This paper is devoted to study the following 3-dimensional full compressible Navier - Stokes equations:

$$
\left\{\begin{array}{l}
\partial_{t} \rho+\operatorname{div}(\rho u)=0 \\
\partial_{t}(\rho u)+\operatorname{div}(\rho u \otimes u)-\mu \triangle u-(\mu+\lambda) \nabla(\operatorname{div} u)+\nabla P=0 \\
c_{v}\left[\partial_{t}(\rho \theta)+\operatorname{div}(\rho \theta u)\right]-\kappa \triangle \theta+P \operatorname{div} u=\frac{\mu}{2}\left|\nabla u+\nabla u^{T}\right|^{2}+\lambda(\operatorname{div} u)^{2}
\end{array}\right.
$$

where $\rho \geq 0$ denotes the density of the mass, $u$ is the velocity.

$$
P=R \rho \theta \quad(a>0, \gamma>1)
$$

is the pressure. $\mu, \lambda, R, c_{v}$ and $\kappa$ are the physical constants satisfying

$$
\mu>0, \lambda+\frac{2}{3} \mu \geq 0, R>0, c_{v}>0, \kappa>0 .
$$

The global existence of classical solutions for the full compressible Navier-Stokes equations was established by Matmusura and Nishida[7, 8] with initial data close to an non-vacuum equilibrium. When the initial density is allowed to vanish, the local existence of strong solutions is recently shown by Cho and Kim[1], which can be described as follows.

Consider the following initial boundary value problem for a viscous heatconductive fluid:

$$
\begin{gathered}
\rho_{t}+\operatorname{div}(\rho u)=0, \text { in }(0, T) \times \Omega \\
(\rho e)_{t}+\operatorname{div}(\rho e u)-\kappa \triangle e+P \operatorname{div} u=Q(\nabla u), \text { in }(0, T) \times \Omega \\
(\rho u)_{t}+\operatorname{div}(\rho u \otimes u)+L u+\nabla P=0, \text { in }(0, T) \times \Omega \\
\left.(\rho, e, u)\right|_{t=0}=\left(\rho_{0}, e_{0}, u_{0}\right), \text { in } \Omega \\
(e, u)=(0,0), \text { on }(0, T) \times \partial \Omega
\end{gathered}
$$

\footnotetext{
*Received October 10, 2009; accepted for publication January 12, 2010.

${ }^{\dagger}$ The Institute of Mathematical Sciences, The Chinese University of Hong Kong, Hong Kong (xdhuang@ustc.edu.cn).
} 


$$
(\rho, e, u)(t, x) \rightarrow\left(\rho^{\infty}, 0,0\right) \text {, as }|x| \rightarrow \infty,(t, x) \in(0, T) \times \Omega .
$$

Here, if $\Omega$ is a bounded domain(or the whole space), then condition (1.8) at infinity (or the boundary condition, respectively) is unnecessary and should be neglected.

Theorem 1.1 (Cho and $\operatorname{Kim}[1]$ ). Let $\rho^{\infty} \in[0, \infty)$ and $q \in(3,6]$ be fixed constants, and define $r$ by

$$
r=2 \text { if } \rho^{\infty}=0, \text { and } r=2 \text { or } 3 \text { if } \rho^{\infty}>0 .
$$

Assume that the data $\rho_{0}, e_{0}, u_{0}$ satisfy the regularity condition

$$
\rho_{0} \geq 0, \quad \rho_{0}-\rho^{\infty} \in W^{1, r} \cap W^{1, q}, \quad\left(e_{0}, u_{0}\right) \in D_{0}^{1} \cap D^{2},
$$

and the compatibility condition

$$
-\kappa \triangle e_{0}-Q\left(\nabla u_{0}\right)=\rho_{0}^{\frac{1}{2}} g_{1} \text { and } L u_{0}+\nabla P_{0}=\rho_{0}^{\frac{1}{2}} g_{2}
$$

for some $\left(g_{1}, g_{2}\right) \in L^{2}$, where $P_{0}=(\gamma-1) \rho_{0} e_{0}$. Then there exist a small time $T_{*}>0$ and a unique strong solution $(\rho, e, u)$ to the initial boundary value problem such that

$$
\begin{aligned}
& \rho-\rho^{\infty} \in C\left(\left[0, T_{*}\right] ; W^{1, r} \cap W^{1, q}\right), \quad \rho_{t} \in C\left(\left[0, T_{*}\right] ; L^{r} \cap L^{q}\right), \\
& (e, u) \in C\left(\left[0, T_{*}\right] ; D_{0}^{1} \cap D^{2}\right) \cap L^{2}\left(0, T_{*} ; D^{2, q}\right), \\
& \left(e_{t}, u_{t}\right) \in L^{2}\left(0, T_{*} ; D_{0}^{1}\right) \text { and }\left(\rho^{\frac{1}{2}} e_{t}, \rho^{\frac{1}{2}} e_{t}\right) \in L^{\infty}\left(0, T_{*} ; L^{2}\right) .
\end{aligned}
$$

REMARK 1.1. We may translate the existence results in terms of the temperature. It is essentially proved that in[1] if

$$
\begin{aligned}
& \inf \rho_{0}>0, \rho_{0} \in W^{1, \tilde{q}}(\Omega) \quad \text { for some } \quad \tilde{q}>N \\
& u_{0} \in H_{0}^{1}(\Omega) \cap H^{2}(\Omega), \theta_{0} \in H^{2}(\Omega), \inf \theta_{0}>0
\end{aligned}
$$

with the following boundary conditions

$$
\left.u\right|_{\partial \Omega}=0,\left.\frac{\partial \theta}{\partial \nu}\right|_{\partial \Omega}=0
$$

where $\nu$ is the normal to $\partial \Omega$.

Then there exist a $T_{*}>0$ and a unique strong solution $(\rho, \theta, u)$ on $\left[0, T_{*}\right]$ to the problem, such that for any $q_{0} \in(N, \tilde{q})$,

$$
\begin{aligned}
& \rho \in C\left(\left[0, T_{*}\right], W^{1, q_{0}}\right), \quad \rho_{t} \in C\left(\left[0, T_{*}\right], L^{q_{0}}\right), \inf \rho>0 \\
& u \in C\left(\left[0, T_{*}\right], H_{0}^{1} \cap H^{2}\right) \cap L^{2}\left(0, T_{*} ; W^{2, q_{0}}\right) \\
& u_{t} \in L^{\infty}\left(0, T_{*} ; L^{2}\right) \cap L^{2}\left(0, T_{*} ; H_{0}^{1}\right) \\
& \theta \in C\left(\left[0, T_{*}\right] ; H^{2}\right) \cap L^{2}\left(0, T_{*} ; W^{2, q_{0}}\right), \theta>0 \\
& \theta_{t} \in L^{\infty}\left(0, T_{*} ; L^{2}\right) \cap L^{2}\left(0, T_{*} ; H^{1}\right)
\end{aligned}
$$

where $N=2$ or 3 .

Concerning this local existence, roughly speaking, for large data, it is still an open problem whether a global small solution exists or not. Even for weak solutions, we mention that only a global "variational solutions" have been obtained by Feiresil[4]. 
However, it is shown in[2] that, with the density away from vacuum, a blow up criterion for the heat-conductive gas is established in two dimensional bounded domain.

Theorem 1.2 (Fan and Jiang[2]). Assume that the initial data satisfy (1.13) (1.14). Let $(\rho, u, \theta)$ be a strong solution of the initial-boundary value problem for 1.1 and satisfy the regularity (1.15). Then, if $T^{*}<\infty$ is the maximal time of existence, then for some $r>2$

$$
\sup _{0 \leq t \leq T}\left(\|\rho\|_{L^{\infty}},\left\|\rho^{-1}\right\|_{L^{\infty}},\|\theta\|_{L^{\infty}}\right)+\int_{0}^{T}\left(\|\rho\|_{W^{1, q_{0}}}+\|\nabla \rho\|_{L^{2}}^{4} d t\right)+\int_{0}^{T}\|u\|_{L^{r, \infty}}^{\frac{2 r}{r-2}} d t=\infty .
$$

Furthermore, if $2 \mu>\lambda$, then

$$
\sup _{0 \leq t \leq T}\left(\|\rho\|_{L^{\infty}},\left\|\rho^{-1}\right\|_{L^{\infty}},\|\theta\|_{L^{\infty}}\right)+\int_{0}^{T}\left(\|\rho\|_{W^{1, q_{0}}}+\|\nabla \rho\|_{L^{2}}^{4} d t\right)=\infty .
$$

REMARK 1.2. The results of Fan and Jiang shows that the density and temperature dominates the regular motion of the fluid.

REMARK 1.3. The main goal of this paper is to show that in fact the gradient of the velocity alone plays a central role in the global existence of strong solutions.

2. Main results. In this paper, we show a certain regularity of $\nabla u$ will be enough to avoid the blow-up of strong solutions.

Basic assumptions: $\mu$ and $\lambda$ are assumed to satisfy the physical restriction

$$
\mu+\frac{3}{2} \lambda \geq 0, \mu>0
$$

and without lose of generality,

$$
c_{v}=1
$$

We shall consider the following initial boundary value problem

$$
\begin{gathered}
\left.u\right|_{\partial \Omega}=0,\left.\quad \frac{\partial \theta}{\partial \nu}\right|_{\partial \Omega}=0 \\
\left.(\rho, u, \theta)\right|_{t=0}=\left(\rho_{0}, u_{0}, \theta_{0}\right) \quad \text { in } \quad \Omega \subset \mathcal{R}^{n}
\end{gathered}
$$

where $n=2,3$, and $\nu$ is the out normal of $\partial \Omega$. Our main theorem is stated as follows.

Theorem 2.1. Let $\Omega \subset R^{3}$ be a bounded domain. $Q_{T}=(0, T) \times \Omega$. Assume that the initial data satisfy (1.13) and (1.14). Let $(\rho, u)$ be a strong solution of the system (1.1) - (1.2) with initial boundary conditions (2.3) and (2.4) satisfying the regularity (1.15). If $T^{*}<\infty$ is the maximal time of existence, then

$$
\lim _{T \rightarrow T^{*}} \int_{0}^{T}\|\nabla u\|_{L^{\infty}(\Omega)}^{2} d t=\infty .
$$

REMARK 2.1. The blow up criterion (2.5) is both sufficient and necessary. 
REMARK 2.2. There is something new in the blowup criteria contrast to $[5,6]$ in the isentropic case. One don't require any restrictions on the viscous coefficients $\mu$ and $\lambda$. The main difficulty is to bound $\|\nabla \rho\|_{L^{\infty} L^{2}}$ at first. In fact, we can derive a $L^{\infty}\left(Q_{T}\right)$ bound of $\theta$ by using the energy equation. The result will be adopted to deduce that the $L^{2}\left(Q_{T}\right)$ norm of the convection term $F=\rho u_{t}+\rho u \cdot \nabla u$ is bounded by that of $\nabla \rho$ as in the isentropic case. This, in turn gives the $L^{\infty} L^{2}$ bound of $\nabla \rho$. Combining the above estimates, one can derive the desired bound for $\|\nabla \rho\|_{L^{\infty} L^{2}}$. The higher estimates on the space and time derivatives of the temperature $\theta$ are also more involved than the non-isentropic case.

Remark 2.3. More recently, Fan[3] told me they obtained a new criteria for the heat-conductive flow, motivated by [5, 6], if $7 \mu>\lambda$, then

$$
\left(\lim _{T \rightarrow T^{*}}\|\theta\|_{L^{\infty}\left(Q_{T}\right)}+\|\nabla u\|_{L^{1} L^{\infty}\left(Q_{T}\right)}\right)=\infty .
$$

In this paper, we denote

$$
L u=\mu \triangle u+(\mu+\lambda) \nabla \operatorname{div} u
$$

the elliptic operator in the momentum equations.

3. Proof of Theorem 2.1. Let $(\rho, u)$ be a strong solution described in Theorem 2.1. We assume that the opposite holds, i.e

$$
\lim _{T \rightarrow T^{*}} \int_{0}^{T}\|\nabla u\|_{L^{\infty}(\Omega)}^{2} d t \leq C<\infty .
$$

By assumption (3.1) and the conservation of mass, the upper and lower bounds of the density follows immediately.

Lemma 3.1. Assume that

$$
\int_{0}^{T}\|\operatorname{divu}\|_{L^{\infty}} d t \leq C, \quad 0 \leq T<T^{*},
$$

then

$$
\left\|\rho, \rho^{-1}\right\|_{L^{\infty}\left(Q_{T}\right)} \leq C, \quad 0 \leq T<T^{*} .
$$

Proof. It follows from the conservation of mass that for $\forall q>1$,

$$
\partial_{t}\left(\rho^{q}\right)+\operatorname{div}\left(\rho^{q} u\right)+(q-1) \rho^{q} \operatorname{div} u=0 .
$$

Integrating (3.4) over $\Omega$ to obtain

$$
\frac{d}{d t} \int_{\Omega} \rho^{q} d x \leq(q-1)\|\nabla u\|_{L^{\infty}(\Omega)} \int_{\Omega} \rho^{q} d x,
$$

i.e

$$
\frac{d}{d t}\|\rho\|_{L^{q}} \leq \frac{q-1}{q}\|\nabla u\|_{L^{\infty}(\Omega)}\|\rho\|_{L^{q}},
$$


which implies immediately

$$
\|\rho\|_{L^{q}}(t) \leq C,
$$

with $C$ independent of $q$, so our lemma follows. The same hold for $\left\|\rho^{-1}\right\|_{L^{\infty}}$.

Lemma 3.2. Assume that

$$
\int_{0}^{T}\|\nabla u\|_{L^{\infty}}^{2} d t \leq C, 0<T<T^{*}
$$

one has

$$
\|\theta\|_{L^{\infty}\left(Q_{T}\right)} \leq C
$$

Proof. Multiplying $\theta^{q+1}$ in the energy equation and integrating gives

$$
\begin{aligned}
& \frac{1}{q+2} \frac{d}{d t} \int_{\Omega} \rho \theta^{q+2} d x-\kappa \int_{\Omega} \triangle \theta \cdot \theta^{q+1}+\int_{\Omega} R \rho \theta^{q+2} \operatorname{div} u d x \\
& =\int_{\Omega}\left[\frac{\mu}{2}\left|\nabla u+\nabla u^{T}\right|^{2}+\lambda(\operatorname{div} u)^{2}\right] \theta^{q+1} d x .
\end{aligned}
$$

Set $f(t)=\int_{\Omega} \rho \theta^{q+2} d x$, one has

$$
\begin{gathered}
\int_{\Omega} R \rho \theta^{q+2} \operatorname{div} u d x \leq C\|\nabla u\|_{L^{\infty}} f(t) \\
\int_{\Omega} \mu\left[\frac{1}{2}\left|\nabla u+\nabla u^{T}\right|^{2}-(\operatorname{div} u)^{2}\right] \theta^{q+1} d x \leq C\|\nabla u\|_{L^{\infty}}^{2} f^{\frac{q+1}{q+2}} \leq C\left(\|\nabla u\|_{L^{\infty}}^{2}+1\right) f(t) .
\end{gathered}
$$

Substituting (3.11)-(3.12) into (3.10), one gets

$$
\partial_{t} f \leq C(q+2)\left(\|\nabla u\|_{L^{\infty}}^{2}+1\right) f .
$$

Hence,

$$
f(t)^{\frac{1}{q+2}} \leq f(0)^{\frac{1}{q+2}} e^{C \int_{0}^{t}\left(\|\nabla u\|_{L \infty}^{2}+1\right) d x} .
$$

Letting $q \rightarrow \infty$, make use of (3.3) yields

$$
\|\theta\|_{L^{\infty}\left(Q_{T}\right)} \leq C
$$

Next, we have $\theta \geq 0$ in $[0, T] \times \Omega$. The proof is standard, one can refer to([4]) for more detail.

Lemma 3.3. Under the condition (3.1), it holds that, for $0 \leq t \leq T<T^{*}$,

$$
\int_{Q_{T}}|\nabla u|^{2}+|\nabla \theta|^{2} d x d t \leq C
$$

Proof. Recalling the entropy estimate, one has

$$
\partial_{t}(\rho s)+\operatorname{div}(\rho s u)-\operatorname{div}\left(\frac{\kappa}{\theta} \nabla \theta\right) \geq \frac{1}{\theta}\left[\frac{\mu}{2}\left|\nabla u+\nabla u^{T}\right|^{2}+\lambda(\operatorname{div} u)^{2}\right]+\frac{\kappa}{\theta^{2}}|\nabla \theta|^{2} .
$$


One can conclude by lemma 3.2 that

$$
\int_{Q_{T}}|\nabla u|^{2}+|\nabla \theta|^{2} d x d t \leq C .
$$

Lemma 3.4. Under the condition (3.1), the following energy estimate holds

$$
\sup _{0 \leq t \leq T} \int_{\Omega} \rho|u|^{2} d x(t)+\int_{Q_{T}}|\nabla u|^{2} d x d t \leq C, 0<T<T^{*} .
$$

Proof. Multiplying $\mathrm{u}$ on both sides of the momentum equations, one gets

$$
\begin{aligned}
& \frac{d}{d t} \int_{\Omega} \rho \frac{|u|^{2}}{2} d x+\int_{\Omega} \mu|\nabla u|^{2}+(\mu+\lambda)(\operatorname{div} u)^{2} d x \\
& =\int_{\Omega} P \operatorname{div} u d x \\
& \leq \frac{\mu}{2} \int_{\Omega}|\nabla u|^{2}+C(\mu) \int_{\Omega} P^{2} d x .
\end{aligned}
$$

This finishes the proof. $\square$

The next lemma shows a connection between a convection term and the gradient of the density.

Lemma 3.5. Let $F=\rho u_{t}+\rho u \cdot \nabla u$. Then it holds that

$$
\int_{Q_{T}} F^{2} d x d t \leq C \int_{Q_{T}}|\nabla \rho|^{2} d x d t+C, \quad 0 \leq T<T^{*} .
$$

Proof. Note that

$$
\int_{Q_{T}} F^{2} d x d t \leq C^{*}\left(\|\rho\|_{L^{\infty}\left(Q_{T}\right)}\right) \int_{Q_{T}} \rho u_{t}^{2} d x d t+2 \int_{Q_{T}}|\rho u \cdot \nabla u|^{2} d x d t .
$$

It follows from lemma 3.1 and 3.4 that

$$
\begin{aligned}
\int_{Q_{T}} F^{2} d x d t & \leq C^{*}\left(\|\rho\|_{L^{\infty}\left(Q_{T}\right)}\right) \int_{Q_{T}} \rho u_{t}^{2} d x d t+\int_{0}^{T}\|\nabla u\|_{L^{\infty}}^{2} \int_{\Omega} \rho^{2} u^{2} d x d t \\
& \leq C \int_{Q_{T}} \rho u_{t}^{2} d x d t+C .
\end{aligned}
$$

Multiplying the momentum equation by $u_{t}$ and integrating show that

$$
\int_{\Omega} \rho u_{t}^{2} d x+\int_{\Omega} \rho u \cdot \nabla u \cdot u_{t} d x+\frac{1}{2} \frac{d}{d t} \int_{\Omega}|\nabla u|^{2} d x=\int_{\Omega} P \operatorname{div} u_{t} d x
$$

the righthand side of (3.23) can be rewritten as

$$
\int_{\Omega} P \operatorname{div} u_{t} d x=\frac{d}{d t} \int_{\Omega} P \operatorname{div} u d x-\int_{\Omega} P_{t} \operatorname{div} u d x
$$


One obtain from the mass equation that

$$
P_{t}+\operatorname{div}(P u)-R \kappa \triangle \theta+R P \operatorname{div} u=R\left[\frac{\mu}{2}\left|\nabla u+\nabla u^{T}\right|^{2}+\lambda(\operatorname{div} u)^{2}\right] .
$$

Consequently

$$
P_{t}=-\left(A_{1}+A_{2}+A_{3}+A_{4}\right),
$$

which can be estimated separately as follows.

$$
\begin{aligned}
\int_{\Omega} A_{1} \operatorname{div} u d x & =\int_{\Omega} P(\operatorname{div} u)^{2}+R \rho u \cdot \nabla \theta \operatorname{div} u+R \theta u \cdot \nabla \rho \operatorname{div} u d x \\
& \leq C\|\operatorname{div} u\|_{L^{2}}^{2}+C\|\rho u\|_{L^{2}}\|\nabla \theta\|_{L^{2}}\|\nabla u\|_{L^{\infty}}+C\|u\|_{L^{2}}\|\nabla \rho\|_{L^{2}}\|\nabla u\|_{L^{\infty}} \\
& \leq C\left(\|\nabla u\|_{L^{2}}^{2}+\|\nabla \rho\|_{L^{2}}^{2}+\|\nabla \theta\|_{L^{2}}^{2}+\|\nabla u\|_{L^{\infty}}^{2}\right) .
\end{aligned}
$$

Note that

$$
\|\nabla P\|_{L^{2}}=R\|\nabla(\rho \theta)\|_{L^{2}} \leq C\|\nabla \theta\|_{L^{2}}+C\|\nabla \rho\|_{L^{2}}
$$

it follows from the elliptic regularity for $L u=F+\nabla P$ that

$$
\begin{gathered}
\|u\|_{H^{2}} \leq C\left(\|F\|_{L^{2}}+\|\nabla P\|_{L^{2}}\right) \\
\int_{\Omega} A_{2} \operatorname{div} u d x=\int_{\Omega} R \kappa \nabla \theta \cdot \nabla \operatorname{div} u d x \\
\leq \epsilon \int_{\Omega} F^{2} d x+C \int_{\Omega}|\nabla \theta|^{2} d x+C \int_{\Omega}|\nabla \rho|^{2} d x \\
\int_{\Omega} A_{3} \operatorname{div} u d x=\int_{\Omega} R P(\operatorname{div} u)^{2} d x \leq C \int_{\Omega}|\nabla u|^{2} d x \\
\int_{\Omega} A_{4} \operatorname{div} u d x \leq C \int_{\Omega}|\nabla u|^{3} d x \\
\leq C\|\nabla u\|_{L^{\infty}}^{2} \int_{\Omega}|\nabla u| d x \\
\leq C \sup _{0 \leq t \leq T}\left(\int_{\Omega}|\nabla u|^{2} d x\right)^{\frac{1}{2}}\|\nabla u\|_{L^{\infty}}^{2} .
\end{gathered}
$$

Direct estimates show that

$$
\begin{aligned}
& \int_{\Omega} P \operatorname{div} u d x(T) \leq \frac{1}{4} \int_{\Omega}|\nabla u|^{2} d x(T)+C \\
& \int_{Q_{T}} \rho u \cdot \nabla u \cdot u_{t} d x d t \leq \frac{1}{2} \int_{Q_{T}} \rho u_{t}^{2}+\int_{Q_{T}} \rho|u \cdot \nabla u|^{2} d x d t \\
& \leq \frac{1}{2} \int_{Q_{T}} \rho u_{t}^{2}+C \int_{0}^{T}\|\nabla u\|_{L^{\infty}}^{2} \int_{\Omega} \rho|u|^{2} d x d t \\
&=\frac{1}{2} \int_{Q_{T}} \rho u_{t}^{2}+C
\end{aligned}
$$


On the other hand, using $F=L u-\nabla P$ again, one obtain

$$
\int_{Q_{T}} P u \cdot \nabla \operatorname{div} u d x d t \leq C \int_{Q_{T}}|\nabla \rho|^{2} d x d t+\epsilon \int_{Q_{T}} F^{2} d x d t+C,
$$

which in turn gives

$$
\begin{aligned}
& \int_{Q_{T}} \rho u_{t}^{2} d x d t+\frac{1}{2} \int_{\Omega}|\nabla u|^{2} d x(T) \\
& \leq C \int_{Q_{T}}|\nabla \rho|^{2} d x d t+2 \epsilon \int_{Q_{T}} F^{2} d x d t+C \sup _{0 \leq t \leq T}\left(\int_{\Omega}|\nabla u|^{2} d x\right)^{\frac{1}{2}}+C .
\end{aligned}
$$

Choosing $\epsilon$ as $2 C^{*} \epsilon<1$, one may conclude

$$
\int_{Q_{T}} F^{2} d x d t \leq C \int_{Q_{T}}|\nabla \rho|^{2} d x d t+C,
$$

which completes the proof of lemma 3.5.

The next lemma will derive the first order spatial derivatives of the density.

Lemma 3.6. Under the condition (3.1), it holds that

$$
\begin{gathered}
\sup _{0 \leq t \leq T} \int_{\Omega}|\nabla \rho|^{2} d x \leq C, 0 \leq T<T^{*}, \\
\int_{Q_{T}} \rho u_{t}^{2} d x d t+\sup _{0 \leq t \leq T} \int_{\Omega}|\nabla u|^{2} d x \leq C \quad, 0 \leq T<T^{*}, \\
\int_{0}^{T}\|u\|_{H^{2}(\Omega)}^{2} d t \leq C, \quad 0 \leq T<T^{*} .
\end{gathered}
$$

Proof. Differentiating the mass equation in (1.1) with respect to $x_{i}$ and multiplying the resulting equation by $2 \partial_{i} \rho$ yield

$$
\partial_{t}\left|\partial_{i} \rho\right|^{2}+\operatorname{div}\left(\left|\partial_{i} \rho\right|^{2} u\right)+\left|\partial_{i} \rho\right|^{2} \operatorname{div} u+2 \partial_{i} \rho \rho \partial_{i} \operatorname{div} u+2 \partial_{i} \rho \partial_{i} u \cdot \nabla \rho=0 .
$$

Integrating over $\Omega$ to show that

$$
\begin{aligned}
\frac{d}{d t} \int_{\Omega}\left|\partial_{i} \rho\right|^{2} d x & =-\int_{\Omega}\left|\partial_{i} \rho\right|^{2} \operatorname{div} u d x-2 \int_{\Omega} \rho \partial_{i} \rho \partial_{i} \operatorname{div} u d x-\int_{\Omega} 2 \partial_{i} \rho \partial_{i} u \cdot \nabla \rho d x \\
& =-\left(A_{1}+A_{2}+A_{3}\right) .
\end{aligned}
$$

Each term on the right hand side of (3.41) can be estimated as follows:

$$
\begin{gathered}
\left|A_{1}(t)\right| \leq\|\operatorname{divu}\|_{L^{\infty}}(t) \int_{\Omega}\left|\partial_{i} \rho\right|^{2} d x \leq\|\operatorname{divu}\|_{L^{\infty}}(t) \int_{\Omega}|\nabla \rho|^{2} d x \\
\left|A_{2}(t)\right| \leq C\|\nabla \rho\|_{L^{2}}\left(\|\nabla P\|_{L^{2}}+\|F\|_{L^{2}}\right) \leq C\left(\int_{\Omega}|\nabla \rho|^{2}+|\nabla \theta|^{2} d x+\int_{\Omega} F^{2} d x\right),
\end{gathered}
$$




$$
\left|A_{3}(t)\right| \leq C\|\nabla u\|_{L^{\infty}}(t) \int_{\Omega}|\nabla \rho|^{2} d x
$$

Consequently,

$$
\frac{d}{d t} \int_{\Omega}|\nabla \rho|^{2} d x \leq C\left(\|\nabla u\|_{L^{\infty}}(t)+1\right) \int_{\Omega}|\nabla \rho|^{2} d x+C \int_{\Omega}\left(F^{2}+|\nabla \theta|^{2}\right) d x .
$$

This, together with Gronwall's inequality yields

$$
\begin{aligned}
\int_{\Omega}|\nabla \rho|^{2} d x(t) & \leq C e^{C \int_{0}^{t}\left(\|\nabla u\|_{\left.L^{\infty}(s)+1\right) d s}\right.}\left(\int_{\Omega}\left|\nabla \rho_{0}\right|^{2} d x\right. \\
& +\int_{0}^{t}\left(\int_{\Omega}\left(F^{2}(s)+|\nabla \theta|^{2}(s) d x\right) e^{-C \int_{0}^{s}\left(\|\nabla u\|_{L} \infty(\tau)+1\right) d \tau} d s\right) \\
& \leq C \int_{0}^{t} \int_{\Omega} F^{2} d x d s+C \\
& \leq C \int_{0}^{t} \int_{\Omega}|\nabla \rho|^{2} d x d s+C .
\end{aligned}
$$

Hence

$$
\sup _{0 \leq t \leq T} \int_{\Omega}|\nabla \rho|^{2} d x \leq C .
$$

Next, it follows from (3.35) and (3.36) that

$$
\int_{Q_{T}} \rho u_{t}^{2} d x d t+\sup _{0 \leq t \leq T} \int_{\Omega}|\nabla u|^{2} d x \leq C .
$$

This, together with $L u=\rho u_{t}+\rho u \cdot \nabla u+\nabla P$ yield

$$
\begin{aligned}
\|u\|_{L^{2}\left(0, T ; H^{2}(\Omega)\right)} & \leq\left\|\rho u_{t}\right\|_{L^{2}\left(Q_{T}\right)}+\|\rho u \cdot \nabla u\|_{L^{2}\left(Q_{T}\right)}+\|\nabla P\|_{L^{2}\left(Q_{T}\right)} \\
& \leq C+C\|\nabla u\|_{L^{2}\left(Q_{T}\right)}+C\|\nabla \rho\|_{L^{2}\left(Q_{T}\right)} \leq C .
\end{aligned}
$$

Next, we show an improved regularity of the temperature.

LEMMA 3.7.

$$
\sup _{0 \leq t \leq T}\|\theta(t)\|_{H^{1}}^{2}+\int_{0}^{T}\left\|\theta_{t}\right\|_{L^{2}}^{2}+\|\theta\|_{H^{2}}^{2} d t \leq C, \quad 0<T<T^{*} .
$$

Proof. Multiplying the energy equation by $\theta_{t}$ and integrating, one may get

$$
\begin{aligned}
\int_{\Omega} \rho \theta_{t}^{2} d x+\frac{\kappa}{2} \frac{d}{d t} \int_{\Omega}|\nabla \theta|^{2} d x & =-\int_{\Omega} P \theta_{t} \operatorname{div} u d x+\int_{\Omega}\left[\frac{\mu}{2}\left|\nabla u+\nabla u^{T}\right|^{2}+\lambda(\operatorname{div} u)^{2}\right] \theta_{t} d x \\
& \leq \frac{1}{2} \int_{\Omega} \rho \theta_{t}^{2} d x+C\|\operatorname{div} u\|_{L^{2}}^{2}+C\|\nabla u\|_{L^{4}}^{4} \\
& \leq \frac{1}{2} \int_{\Omega} \rho \theta_{t}^{2} d x+C\|\operatorname{div} u\|_{L^{2}}^{2}+C\|\nabla u\|_{L^{\infty}}^{2} \int_{\Omega}|\nabla u|^{2} d x \\
& \leq \frac{1}{2} \int_{\Omega} \rho \theta_{t}^{2} d x+C\|\operatorname{div} u\|_{L^{2}}^{2}+C\|\nabla u\|_{L^{\infty}}^{2} .
\end{aligned}
$$


Recall that

$$
\kappa \triangle \theta=\rho \theta_{t}+\rho u \cdot \nabla \theta+P \operatorname{div} u-\frac{\mu}{2}\left[\left|\nabla u+\nabla u^{T}\right|^{2}-(\operatorname{div} u)^{2}\right] \in L^{2}\left(Q_{T}\right) .
$$

This finishes the proof of lemma 3.7.

LEMma 3.8. Under the condition (3.1), it holds that

$$
\begin{gathered}
\sup _{0 \leq t \leq T}\left\|\rho^{1 / 2} u_{t}(t)\right\|_{L^{2}}^{2}+\int_{Q_{T}}\left|\nabla u_{t}\right|^{2} d x d t \leq C, \quad 0 \leq T<T^{*} . \\
\sup _{0 \leq t \leq T}\|u\|_{H^{2}} \leq C, \quad 0 \leq T<T^{*} .
\end{gathered}
$$

Proof. Differentiating the momentum equations in (1.1) with respect to time $t$ yields

$$
\rho u_{t t}+\rho u \cdot \nabla u_{t}-\triangle u_{t}+\nabla p_{t}=-\rho_{t}\left(u_{t}+u \cdot \nabla u\right)-\rho u_{t} \cdot \nabla u .
$$

Taking the inner product of the above equation with $u_{t}$ in $L^{2}(\Omega)$ and integrating by parts, one gets

$$
\begin{aligned}
& \frac{d}{d t} \int_{\Omega} \frac{1}{2} \rho u_{t}^{2} d x+\int_{\Omega}\left|\nabla u_{t}\right|^{2} d x-\int_{\Omega} P_{t} \operatorname{div} u_{t} d x \\
& =-\int_{\Omega}\left(\rho u \cdot \nabla\left[\left(u_{t}+u \cdot \nabla u\right) u_{t}\right]+\rho\left(u_{t} \cdot \nabla u\right) \cdot u_{t}\right) d x .
\end{aligned}
$$

The last term on the left-hand side of (3.55) can be rewritten as (using (2.33)): It follows from (3.56) and (3.57) that

$$
\begin{aligned}
& \frac{d}{d t} \int_{\Omega} \frac{1}{2} \rho u_{t}^{2} d x+\int_{\Omega}\left|\nabla u_{t}\right|^{2} d x-\int_{\Omega} P_{t} \operatorname{div} u_{t} d x \\
& \leq \int_{\Omega}\left(2 \rho|u|\left|u_{t}\right|\left|\nabla u_{t}\right|+\rho|u|\left|u_{t}\right||\nabla u|^{2}+\rho|u|^{2}\left|u_{t}\right|\left|\nabla^{2} u\right|+\rho|u|^{2}|| \nabla u|| \nabla u_{t} \mid\right. \\
& \left.+\rho\left|u_{t}\right|^{2}|\nabla u|+|\nabla P||u|\left|\nabla u_{t}\right|+\gamma P|u||\nabla u|\left|\nabla^{2} u\right|+\gamma^{2} P|\nabla u|^{3}\right) d x \\
& \equiv \sum_{i=0}^{8} F_{i} .
\end{aligned}
$$

Recall Lemmas 3.6-3.7 that

$$
\left\|P_{t}\right\|_{L^{2}\left(Q_{T}\right)}=\left\|R \rho_{t} \theta+R \rho \theta_{t}\right\|_{L^{2}\left(Q_{T}\right)} \leq C
$$

which gives

$$
\int_{\Omega} P_{t} \operatorname{div} u_{t} d x \leq \epsilon \int_{\Omega}\left|\nabla u_{t}\right|^{2} d x+C(\epsilon) .
$$

Now, we estimate each $F_{i}$ separately. 


$$
\begin{aligned}
\left|F_{1}\right| & =\int_{\Omega} 2 \rho\left|u \| u_{t}\right|\left|\nabla u_{t}\right| d x \\
& \leq C\|u\|_{L^{6}}\left\|\rho^{1 / 2} u_{t}\right\|_{L^{3}}\left\|\nabla u_{t}\right\|_{L^{2}} \\
& \leq C\left\|\rho^{1 / 2} u_{t}\right\|_{L^{2}}^{\frac{1}{2}}\left\|\nabla u_{t}\right\|_{L^{2}}^{\frac{3}{2}} \\
& \leq \epsilon\left\|\nabla u_{t}\right\|_{L^{2}}^{2}+C\left\|\rho^{1 / 2} u_{t}\right\|_{L^{2}}^{2} .
\end{aligned}
$$

Thus, it follows from Hölder inequality, Sobolev imbedding and interpolation inequality that

$$
\begin{aligned}
& \left|F_{2}\right|=\int_{\Omega} \rho|u|\left|u_{t}\right||\nabla u|^{2} d x \\
& \leq C\|u\|_{L^{6}}\left\|u_{t}\right\|_{L^{6}}\|\nabla u\|_{L^{3}}^{2} \\
& \leq C\left\|\nabla u_{t}\right\|_{L^{2}}\|\nabla u\|_{L^{2}}\|\nabla u\|_{L^{6}} \\
& \leq C\left\|\nabla u_{t}\right\|_{L^{2}}\|\nabla u\|_{L^{6}} \\
& \leq \epsilon\left\|\nabla u_{t}\right\|_{L^{2}}^{2}+C\|u\|_{H^{2}}^{2} \text {, } \\
& \left|F_{3}\right|=\int_{\Omega} \rho|u|^{2}\left|u_{t}\right|\left|\nabla^{2} u\right| d x \\
& \leq\left\|u^{2}\right\|_{L^{3}}\left\|u_{t}\right\|_{L^{6}}\left\|\nabla^{2} u\right\|_{L^{2}} \\
& \leq \epsilon\left\|\nabla u_{t}\right\|_{L^{2}}^{2}+C\|u\|_{H^{2}}^{2} \text {, } \\
& \left|F_{4}\right|=\int_{\Omega} \rho|u|^{2}|\nabla u|\left|\nabla u_{t}\right| d x \\
& \leq C\left\|\nabla u_{t}\right\|_{L^{2}}\|\nabla u\|_{L^{6}}\left\|u^{2}\right\|_{L^{3}} \\
& \leq C\|\nabla u\|_{L^{6}}\left\|\nabla u_{t}\right\|_{L^{2}} \\
& \leq \epsilon\left\|\nabla u_{t}\right\|_{L^{2}}^{2}+C\|u\|_{H^{2}}^{2} \text {, } \\
& \left|F_{5}\right|=\int_{\Omega} \rho\left|u_{t}\right|^{2}|\nabla u| d x \\
& \leq C\left\|\rho u_{t}^{2}\right\|_{L^{2}}\|\nabla u\|_{L^{2}} \\
& \leq C\left\|\rho^{1 / 2} u_{t}\right\|_{L^{4}}^{2} \\
& \leq \epsilon\left\|u_{t}\right\|_{L^{6}}^{2}+C\left\|\rho^{1 / 2} u_{t}\right\|_{L^{2}} \text {, } \\
& \left|F_{6}\right|=\int_{\Omega}|\nabla P||u|\left|\nabla u_{t}\right| d x \\
& \leq C\|\nabla P\|_{L^{2}}\|u\|_{L^{\infty}}\left\|\nabla u_{t}\right\|_{L^{2}} \\
& \leq C\|u\|_{H^{2}}\left\|\nabla u_{t}\right\|_{L^{2}} \\
& \leq \epsilon\left\|\nabla u_{t}\right\|_{L^{2}}^{2}+C\|u\|_{H^{2}}^{2} \quad
\end{aligned}
$$




$$
\begin{aligned}
\left|F_{7}\right| & =\int_{\Omega} \gamma P|u||\nabla u|\left|\nabla^{2} u\right| d x \\
& \leq C\left\|\nabla^{2} u\right\|_{L^{2}}\|\nabla u\|_{L^{2}}\|u\|_{L^{\infty}} \\
& \leq C\left\|\nabla^{2} u\right\|_{L^{2}}\|u\|_{L^{\infty}} \\
& \leq C\|u\|_{H^{2}}^{2}+C, \\
\left|F_{8}\right| & =\int_{\Omega} \gamma^{2} P|\nabla u|^{3} d x \\
& \leq C \int_{\Omega}|\nabla u|^{3} d x \\
& \leq C\|\nabla u\|_{L^{\infty}(\Omega)} \int_{\Omega}|\nabla u|^{2} d x \\
& \leq C\|\nabla u\|_{L^{\infty}(\Omega)} .
\end{aligned}
$$

Collecting all the estimates for $F_{i}$, we conclude that

$$
\begin{aligned}
& \frac{d}{d t} \int_{\Omega}\left(\frac{1}{2} \rho u_{t}^{2}+\frac{\gamma}{2} p(\operatorname{div} u)^{2}\right) d x+\int_{\Omega}\left|\nabla u_{t}\right|^{2} d x \\
& \leq 5 \epsilon \int_{\Omega}\left|\nabla u_{t}\right|^{2} d x+C\left(\left\|\rho^{1 / 2} u_{t}\right\|_{L^{2}}^{2}+\|u\|_{H^{2}}^{2}+\|\nabla \rho\|_{L^{2}}^{2}+\|\nabla u\|_{L^{\infty}}\right) .
\end{aligned}
$$

Therefore, taking $\epsilon$ small enough in (3.67) yields

$$
\sup _{0 \leq t \leq T}\left\|\rho^{1 / 2} u_{t}(t)\right\|_{L^{2}}^{2}+\int_{Q_{T}}\left|\nabla u_{t}\right|^{2} d x d t \leq C, \quad 0 \leq T<T^{*} .
$$

Moreover,

$$
\begin{aligned}
\|u\|_{H^{2}} & \leq C\left(\left\|\rho^{\frac{1}{2}} u_{t}\right\|_{L^{2}}+\|u\|_{L^{6}}\|\nabla u\|_{L^{3}}+\|\nabla P\|_{L^{2}}\right) \\
& \leq C\left(\left\|\rho^{\frac{1}{2}} u_{t}\right\|_{L^{2}}+\|\nabla u\|_{L^{2}}^{\frac{3}{2}}\|u\|_{H^{2}}^{\frac{1}{2}}+\|\nabla P\|_{L^{2}}\right) .
\end{aligned}
$$

Therefore,

$$
\sup _{0 \leq T<T^{*}}\|u\|_{H^{2}}^{2} \leq C
$$

Furthermore, the following lemma gives bounds of spatial derivatives of the density and the second spatial derivatives of the velocity.

Lemma 3.9. Under the condition (3.1), let $q_{0}$ be as the same in Theorem 1.1. Then it holds that

$$
\begin{gathered}
\sup _{0 \leq t \leq T}\left(\left\|\rho_{t}(t)\right\|_{L^{q_{0}}}+\|\rho\|_{W^{1, q_{0}}}\right) \leq C, \quad 0 \leq T<T^{*}, \\
\int_{0}^{T}\|u(t)\|_{W^{2, q_{0}}}^{2} d t \leq C, \quad 0 \leq T<T^{*}, q_{0}=\min (6, \tilde{q}) .
\end{gathered}
$$


Proof. It follows from (3.68) and (3.69) that

$$
\begin{gathered}
u_{t} \in L^{2}\left(0, T ; L^{6}(\Omega)\right), \nabla u \in L^{6}\left(Q_{T}\right), \\
F \in L^{2}\left(0, T ; L^{6}(\Omega)\right), \nabla P \in L^{2}\left(0, T ; L^{6}(\Omega)\right) .
\end{gathered}
$$

Differentiating the mass equation in (1.1) with respect to $x_{i}$, and multiplying the resulting identity by $q_{0}\left|\partial_{i} \rho\right|^{q_{0}-2} \partial_{i} \rho$, one gets after integration that

$$
\begin{aligned}
\frac{d}{d t} \int_{\Omega}\left|\partial_{i} \rho\right|^{q_{0}} d x & =-\left(q_{0}-1\right) \int_{\Omega}\left|\partial_{i} \rho\right|^{q_{0}} \operatorname{div} u d x-q_{0} \int_{\Omega} \rho\left|\partial_{i} \rho\right|^{q_{0}-2} \partial_{i} \rho \partial_{i} \operatorname{div} u d x \\
& -q_{0} \int_{\Omega}\left|\partial_{i} \rho\right|^{q_{0}} \partial_{i} \rho \partial_{i} u \cdot \nabla \rho d x \\
& =-\left(B_{1}+B_{2}+B_{3}\right) .
\end{aligned}
$$

Each quantity in the righthand side of (3.71) can bounded as follows.

$$
\begin{gathered}
\left|B_{1}(t)\right| \leq C\|\nabla u\|_{L^{\infty}}(t) \int_{\Omega}\left|\partial_{i} \rho\right|^{q_{0}} d x \leq C\|\nabla u\|_{L^{\infty}}(t) \int_{\Omega}|\nabla \rho|^{q_{0}} d x \\
\left|B_{2}(t)\right| \leq C\left\||\nabla \rho|^{q_{0}}\right\|_{L^{\frac{q_{0}}{q_{0}}-1}}\left(\|\nabla P\|_{L^{q_{0}}}+\|F\|_{L^{q_{0}}}\right) \\
\left|B_{3}(t)\right| \leq C\|\nabla u\|_{L^{\infty}}(t) \int_{\Omega}|\nabla \rho|^{q_{0}} d x .
\end{gathered}
$$

Substituting (3.71)-(3.73) into (3.74), one has

$$
\frac{d}{d t}\|\nabla \rho\|_{L^{q_{0}}} \leq C\left(\|\nabla u\|_{L^{\infty}}(t)+1\right)\|\nabla \rho\|_{L^{q_{0}}}+C\|F\|_{L^{q_{0}}} .
$$

Hence,

$$
\sup _{0 \leq t \leq T}\|\nabla \rho\|_{L^{q_{0}}} \leq C
$$

Therefore, due to this, (3.71) and interpolation inequality, one has

$$
\rho_{t}=-(u \cdot \nabla \rho+\rho \operatorname{div} u) \in L^{\infty} L^{q_{0}} .
$$

Finally, taking into account that

$$
L u=F+\nabla P \in L^{2} L^{q_{0}},
$$

one has

$$
\int_{0}^{T}\|u\|_{W^{2, q_{0}(\Omega)}}^{2} d t \leq C
$$

This finishes the proof of lemma 3.9.

We will improve the regularity of the temperature $\theta$. 
LEMMA 3.10.

$$
\sup _{0 \leq t \leq T}\left\|\theta_{t}\right\|_{L^{2}}^{2}+\left\|\nabla \theta_{t}\right\|_{L^{2}\left(Q_{T}\right)}^{2} \leq C, \quad 0<T<T^{*} .
$$

Proof. Differentiating the energy equation with the time $t$, one gets

$$
\begin{aligned}
& \frac{1}{2} \frac{d}{d t} \int_{\Omega} \rho \theta_{t}^{2} d x+\kappa \int_{\Omega}\left|\nabla \theta_{t}\right|^{2} d x \\
& \leq \int_{\Omega} P\left|\operatorname{div} u_{t}\right|\left|\theta_{t}\right|+R\left|\rho_{t}\right||\theta \operatorname{div} u|\left|\theta_{t}\right|+R \rho|\operatorname{div} u|\left|\theta_{t}\right|^{2} \\
& +2 \mu|\nabla u|\left|\nabla u_{t}\right|\left|\theta_{t}\right|+\left|\rho_{t}\right||u||\nabla \theta|\left|\theta_{t}\right|+\rho\left|u_{t}\right||\nabla \theta|\left|\theta_{t}\right|+\left|\rho_{t}\right|\left|\theta_{t}\right|^{2} d x \\
& =\sum_{i=1}^{7} B_{i} .
\end{aligned}
$$

We can estimate each $B_{i}$ as follows

$$
\begin{gathered}
\left|B_{1}\right| \leq C\left\|\nabla u_{t}\right\|_{L^{2}}\left\|\theta_{t}\right\|_{L^{2}} \leq C\left(\left\|\nabla u_{t}\right\|_{L^{2}}^{2}+\left\|\theta_{t}\right\|_{L^{2}}^{2}\right), \\
\left|B_{2}\right| \leq\left\|\rho_{t}\right\|_{L^{2}}\|\operatorname{div} u\|_{L^{\infty}}\left\|\theta_{t}\right\|_{L^{2}} \leq C\left(\|\nabla u\|_{L^{\infty}}^{2}+\left\|\theta_{t}\right\|_{L^{2}}^{2},\right. \\
\left|B_{3}\right| \leq C\|\operatorname{div} u\|_{L^{3}}\left\|\theta_{t}\right\|_{L^{3}}^{2} \leq C\left\|\theta_{t}\right\|_{L^{2}}\left\|\theta_{t}\right\|_{L^{6}} \leq \epsilon\left\|\nabla \theta_{t}\right\|_{L^{2}}^{2}+C(\epsilon)\left\|\theta_{t}\right\|_{L^{2}}^{2}, \\
\left|B_{4}\right| \leq C\left\|\nabla u_{t}\right\|_{L^{2}}\left\|\theta_{t}\right\|_{L^{6}} \leq C\left\|\nabla u_{t}\right\|_{L^{2}}\left\|\theta_{t}\right\|_{L^{2}}+C\left\|\nabla u_{t}\right\|_{L^{2}}\left\|\nabla \theta_{t}\right\|_{L^{2}}, \\
\left|B_{5}\right| \leq C\left\|\rho_{t}\right\|_{L^{2}}\|\nabla \theta\|_{L^{4}}\left\|\theta_{t}\right\|_{L^{4}}, \\
\left|B_{6}\right| \leq C\left\|u_{t}\right\|_{L^{6}}\|\nabla \theta\|_{L^{2}}\left\|\theta_{t}\right\|_{L^{6}}, \\
\left|B_{7}\right| \leq C\left\|\rho_{t}\right\|_{L^{2}}\left\|\theta_{t}\right\|_{L^{4}}^{2} \leq C\left\|\theta_{t}\right\|_{L^{4}}^{2} \leq C\left\|\theta_{t}\right\|_{L^{2}}\left\|\theta_{t}\right\|_{L^{6}} .
\end{gathered}
$$

Collecting all the estimates (3.80-3.86) and applying Lemmas 3.6-3.10, we easily obtain

$$
\sup _{0<T<T^{*}}\left\|\theta_{t}\right\|_{L^{2}}^{2}+\int_{0}^{T}\left\|\theta_{t}\right\|_{H^{1}}^{2}+\|\theta\|_{H^{2}}^{2} d t \leq C
$$

Finally, the following lemma gives the desired estimated for the temperature. 
Lemma 3.11. Under the condition (3.1), it holds that

$$
\sup _{0 \leq t \leq T}\|\theta\|_{H^{2}} \leq C, \quad 0<T<T^{*}
$$

Proof. We may rewrite the energy equation as

$$
\kappa \triangle \theta=c_{v}\left[\partial_{t}(\rho \theta)+\operatorname{div}(\rho \theta u)\right]+P \operatorname{div} u-\left(\frac{\mu}{2}\left|\nabla u+\nabla u^{T}\right|^{2}+\lambda(\operatorname{div} u)^{2}\right) .
$$

Based on the lemmas (3.6-3.10), one immediately has (3.88) by noticing that the righthand side of (3.89) is bounded in $L^{\infty} L^{2}$.

We are now ready to extend the strong solutions beyond the time $T^{*}$.

In fact, Lemmas 3.6-3.8 and Lemma 3.11, the functions $\left.(\rho, u, \theta)\right|_{t=T^{*}}=$ $\lim _{t \rightarrow T^{*}}(\rho, u, \theta)$ satisfy the conditions imposed on the initial data $(1.13)-(1.14)$ at the time $t=T^{*}$. Therefore, one can take $\left.(\rho, u, \theta)\right|_{t=T^{*}}$ as the initial data and apply the local existence Theorem 1.1 to extend our local strong solution beyond $T^{*}$. This contradicts the assumption on $T^{*}$.

Acknowledgement. This work is done in the Ph.D period of the author in the Chinese University of Hong Kong. The author would like to thank the referees for very helpful advices on improvements.

\section{REFERENCES}

[1] Y. Cho And H. Kim, Existence results for viscous polytropic fluids with vacuum, J. Differential Equations, 228 (2006), pp. 377-411.

[2] J. FAN AND S. JiAng, Blow-Up criteria for the navier-stokes equations of compressible fluids, J. Hyper. Diff. Equa., 5:1 (2008), pp. 167-185.

[3] J. FAn, S. JIANG AND Y. OU, a blow-up criteria for compressible viscous heat-conductive flows, Ann.I.H. Poincare (2009), doi:10.1016/j.anihpc.2009.09.012.

[4] E. FeIReisl, Dynamics of viscous compressible fluids Oxford University Press, 2004.

[5] X. Huang and Z. XIn, A Blow-up criterion for the compressible Navier-Stokes equations, arXiv:0902.2606.

[6] X. Huang and Z. Xin, A Blow-Up Criterion for Classical Solutions to the Compressible Navier-Stokes Equations, arXiv:0903.3090.

[7] A. Matsumura and T. Nishida, Initial-boundary value problems for the equations of motion of compressible viscous and heat-conductive fluids, Comm. Math. Phys., 89 (1983), pp. 445464.

[8] A. Matsumura AND T. Nishida, Initial-boundary value problems for the equations of motion of compressible viscous and heat-conductive fluids, Comm. Math. Phys., 89 (1983), pp. 445464. 
X. HUANG 